A major purpose of the lechnical Information Center is to provide the broadest dissemination possible of information contained in DOE's Research and Development Reports to business, industry, the academic community, and federal, state and local governments.

Although a small portion of this report is not reproducible, it is being made available to expedite the availability of information on the research discussed herein. 
CA-UR-36-3744

:TLE: "ANALYSIS OF THE hUMAN ELECTROENCE?HALOGRAM WITH METHODS FROM NONLINEAR DYNAMICS"

LA-UR- $-86-3744$

DE87 002928

AUTHOA(S): GOTTFRIED MAYER-KFrSS

JOACHIM HOLZFUSS

SUBMITTED TO. TEMPORAL DISORDER IN HUMAN OSCILLATORY SYSTEMS

\section{DISCLAIMER}

This roport was propared as an eccount of wort eponerod by an agency of the United State Government. Neither the United Stutes Governmont nor any aconcy thereof, nor eny of thair employees, makee any warcanty, expreses or Impliod, or asumes any logal liability or raposad. blitily for the eccuracy, completeneed, or usofulneas of any Information, app aratus, product, or process disctosed, or represents that ite use would not infringe privalely owned righe. Rofor-

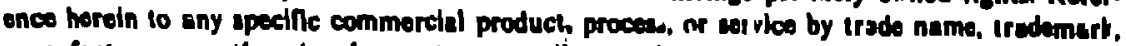
manufacturer, of otherwise does not necosarily conatilute of imply its endonoment, recominondation, or favoring by the Uniled Sintes Government or any asency thereof. The viem and opintons of uuthon expresed herein do not necesandly stale or reflect thowe of the United State Clovernment of any agency thereof. 


\title{
Analysis of the Human Electroencephalogram with Methods from Nonlinear Dynamics
}

\author{
Gottfried Mayer-Kress ${ }^{1,2}$ and Joacinim Holzfuss ${ }^{1,3}$ \\ 1 Center for Nonlinear Studies, Los Alamos National Laboratory, Los Alamos, NM 87545, USA \\ 2 Observatoire de Nire, Nice, France \\ ${ }^{3}$ 3. Phys. Inst., Univ. Goettingen, D-3400 Goeitingen, W'.-Germany
}

\begin{abstract}
:
We apply several different methods from nonlinear dynamical systems to the analysis of the degree of temporal disorder in data from human EEG. Among these are methods of geometrical reconsiruction, dimensional complexity, mutual information content, and two different approaches for estimating Lyapunov characteristic exponents. We show how the naive interpretation of numerical results can lead to a considrrable underestimation of the dimensional complexity. This is true even when the errors from least squares fits are small. We present more realistic error estimates and show that they seem to contain additionai, impoitant information. By applying independent methods of analysis to the same data sets for a given lead, we find that the degree of temporal disorder is minimal in a "resting awaken state and increases in sleep as well as in fluroxene induced general anesthesia. At the same time the siatistical errors appear to decrease, which can be interpretated as a transition to a more uniform dynamical state.
\end{abstract}

\section{Introduction:}

For more than sixty years it is known that the human brain exhibits elect:o-magnetic activity which can be recorded externally as electro- or magneto-encephalogram (EEG or MEG).

The microscopic genesis of the EEG is not understood [1]. Macroscopically, electrical signals appear to originate from extensive dendritic arborizations on neurons. It is in this extensive overlap that excitations within one dendritic tree are conveyed to the arborizations of adjacent neurons $\mid 2$. These collective excitations, in turn, generate signals that are coordinated in space and time near the surface of the brain. Beyond this, there is some attenuation and, what is more important, a filtering of the brain's electrical activity by the intervening skull and scalp. A detailed discussion of the clinical problems related to the EEG recording and possible error sources which are related to artefacts has been given elsewhere [3].

It is known for a long time that the electrical signal of the EEG is in some way related to the inental activity of the brain. So, for iustance it is possible for trained individuals to distinguish between sleep and awake states by visually inspecting th: EEG. Thus there have many attempts to finc some quantitative observables which could measure these changes $[4 \mid$. A limited success w'ils achieved by fourier analyzing the EEG signal and specifying the different mental states according to the distribution of the power of the signal in different frequency bands. The most famous among them is the frequency band around $10 \mathrm{~Hz}$ (alpha waves) which is in some way related to a relaxed state with eyes closed. The implicit assumption in this and similar ways of analysis is that the "active modes" in the brain which produce the elertro-magnetic signal, are linear periodic oscillators. It is known, nowever that the number of these fourier modes can be much larger than the number of modes which actually are responsible for the physical, or in this case, bic-chemical processes $[5]$.

Thus, while spectral methods, which analyze frequency bands are optimized for regular periodic or quast-periodic signals, the applicability of these methods becomes very limited in cases where the signal is intrinsically very irregular without very sharp and well defined frequency bande. This situation of deterministic chaos is known to be fairly common in nonlinear dynanical systems and is disclissed as an origin of many biological and clinical cases of temporal disorders $|6|$.

Thercfore it appears to be of greal importance to develor new methods for characterizing hiological temporal signals. This should make it possible to classify them according to their degree. It of coinplexity or a:cording to the number of nonlinear modes which are generating them. 


\section{Geometrical Reconstruction:}

The main feature of this technique is to reinterprete time-signals as multidimensional geometrical objects [7]. This allows us to use notions from geometry to describe and characterize a time evolution. With methods based on these principles we obtain information which is in principle independent from the one we gain from the classical approaches using autocorrelation functions and power spectra. These are related via Fourier transformation, and therefore express the same kind of information about the presence of periodic oscillators in the signal.

The geometrical view of dynamical processes is based on the assumption that signals are generated by some deterministic and finite dimensional (generically nonlinear) dynamical system, which is not necessarily the superposition of periodic oscillators. The observed signal can then be interpreted as the projection of a multidimensional phase-space trajectory.

In the following analysis we reconstruct time-delayed vectors from EEG data with increasing values of the "embedding dimension" $N$, which specifies the number of components of the reconstructed vectors or the number of time delays. The general form of such a phase-space vector is given by:

$$
\vec{x}_{N}(t)=(x(t), x(t+\tau), x(t+2 \tau), \ldots, x(t+(N-1) \tau))
$$

These reconstructed phase-trajectories for EEG data can be plotted in different projections for different time delays and might be helpful in recognizing specific features in the data which are not so evident in the time series (see e.g. $[3,8]$ for some examples). These objects in phase space (sometimes called "attractors") are the starting point for the following analysis.

In the next section, we give a quick review of the concept of "mutual information content" as a convenient tool for finding an optimal choice for the time delay $\tau$.

\section{Mutual Information Content:}

In order to get a satisfactory reconstruction from time-series data, we require that the components $x\left(t+k^{+}\right)$of the vector $\vec{x}_{N}(t)$ be independent. The first idea that comes to mind concerning this optimum, is to use a time delay for which the autocorrelation function of the time-series vanishes, i.e., the two coordinate are "uncorrelated". In the present context of nonlinear systerns. however, this intuition might be misleading. There exist examples of low dimensionai deterministic systems which possess a power-spectrum which is identical with that of random noise [9].

Thus the autocorrelation function cannot distinguish between a purely deterministic process, where the dynamics is generated by just a few nonlinear modes and random noise, which represents a system where infinitely many (linear) modes are contributing to the dynamics.

SHAW [10] studied this problem from an information theoretical point of view, asking ine question: Assume we know the result $x(t)$ of a measurement at time $t$. Linder real experimental conditions with nois, and finite resolution. what can be predicted about the outcome of the same measurement at time $T$ later? FRASER and SWINNEY 11 l developed this idea further and applied it to various experimental situations. By using the "mutual information content" as the criterion for choosing $\tau$, rather than auto-crrelation, we are able to obtain a better reconstruction in the sence that more details will become distinguishable at a given resolution.

Using codes developed by FRASER |11|, which calculate mutual information content (MIC), we determine the "optimal" choice of time delay $\tau$ from a string of $\geq 4096$ data-points and its copy shifted by $\tau$. In general, we choose $\tau$ where the MIC has its first minimum. For some EEG data, lowever, there exists no distinct first minimum. In these instances. We choose a value of $r$ which is comparable to delays used in the other data sets. We find that the results are relatively stable within fuirly large ranges of $\tau$.

In the following section, we present the basic ideas behind dimensionel analysis without going into the formalism that is reviewed in $|12|$.

\section{Dimensional Analysis:}

In the strict sense, the dimension of a dynamical system is a measure of the number of independent variables needed to specify its state at a given instant and therefore, it is also a measure of complexity |13!. A method introduced by P.GRASSBERGER and I. PROCACCIA (we shall refer to it as "G-P"), which requires minimal computer time and memory, is now in wide use ! 14 !: Here 
of the algorithms and a discussion of their reliability and error estimates are reviewed in [15]. A fast implementation of the code is also described in [16].

The original idea of G-P was to calculate how the number $n(r)$ of pairs of vectors $\vec{x}_{:}=\vec{x}_{N}\left(t_{i}\right)$, which are separated by distances less than $r$, changes as a function of $r$. They call this quantity "correlation integral" and in the general "ase it should scale like:

$$
n(r)=r^{d}
$$

where $d$ is the "correlation" dimension of the set. The way it is usually obtained is by taking the logarithm which yields the linear function:

$$
\log n(r)=d \log (r)
$$

Thus if we plot the dimension curve $\log n(r)$ vs. $\log (r)$ we expect to observe a straight iine, where the slope $d$ corresponds to the dimension of the set. In practice, this method will never result in a perfectly straight line over the entire region $\log ($.$) , because of the finite size of the vectors and$ other effects which are discussed in [18]. However, there is usually a smaller region over which a "straight line" may be fitted and therefore, a slope can be read off which is then called "dimension".

In our opinion, a major drawback of this algorithm is that by fitting a straight line to a portion of the dimension curve, it is always possible to get a result, namely a slope $d$, but it is not trivial to decide whether this value is really significazt or purely due to artifacts.

After calculating time-delayed coordinates from normalized EEG data, we reconstruct phase space vectors $\bar{x}_{N}\left(t_{i}\right)$ for increasing embedding dimensions $N=1,2, \ldots, 20$. However, to use the original G-P algorithm, which calculates distances between all pairs $\left(\vec{x}_{N}\left(t_{2}\right), \vec{x}_{N}(t),\right)$ of data points, would be too time consuming. Therefore, we choose a smaller set of $n_{\text {ref }}$ equally spaced (in time) reference points $\bar{x}_{N}\left(t_{k}\right)$ and compute distances between reference points and the rest of the data points. Typically, we use 200 reference points and 3,000-20,000 data points.

To compute the correlation dimension we determine for each value of $\log (r)$ the average number $\log n(r)$ of pairs which are separated by $\dot{r} \leq r$. The error bars in fig. 1 indicate the width of this distribution for each discrete value of $\log (r)$. In $[15]$ we showed how this method can produce systematically small values for the correlation dimension in the case of finite data sets from strongly non-uniform attractors. These error estimates indicate the maximal variations in slope for line segments in the "scaling region." For each data set, the overall error is proportional to the average size of the error-bars in the dimension curve and inversely proportional to the length of the "scaling region." Therefore, fitting over large "scaling regions" with large error tars yields similar results to fitting over small "scaling regions" with small error bars (see 15) for details). In the literature, errors based on a least squares fit are often quoted. We think this is far too optimistic. especially for small scaling regions.

If we look at the dimension-curves for each individual reference point, we see that many of them are displaced along the horizontal axis due to variations in scale as described in $\mid 15)$. Their slopes correspond to the "point-wise dimension" of the different reference points and their average determines the "information dimension" $D^{(1)}|13|$ (see fig. 3). In the case of a vrry large number of data points the averaging according to the correlation dimension $D^{(2)}$ would yivild a result which gets close to $D^{(1)}$. W'ith our EEG data, however, we find in the case of "low dimunsional chaos" (awake, quiet, eyes closed, sree fig.1) a value for the G-P dimensior, of $D^{(2)}=4.3 \pm 2.2$. For the same data set we obtain $D^{(1)}=6.4 \pm 1.2$ (see fig.4). In fig. 5 we show the frequency distribution of the measured values of the point-wise dimension of the set of $n_{r e f}=200$ reference points. The errors indicated in these plots are statistical and more reliable. They also seem to be consistently smaller. When the patient is under fluroxene induced genera! anesithesia the G.P' dimension increases to $D^{(2)}=8.0 \pm 3.8|3|$. This uncertainty of $50 \%$ at such a high value for $D^{(2)}$ is very well compatible with the hypothesis of noise. For this data set we find for the information dimension $D^{(1)}=7.1 \pm 0.5$ (fig. 6). Thus we see a consistent increase in the dimcnsional complexity under the influence of fluroxene. Besides that it seems that the fluctuations in the dimension values have, decreased and the behavior has become more uniform (fig.7). We shall see below that this feature is also reflected in the observed Lyapunov exponents.

Similar behavior is observed in the sleep data, but the effect is not so pronounced there. We

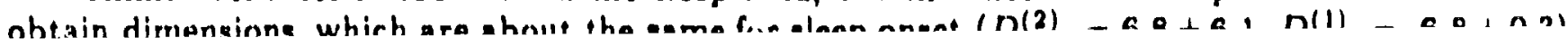




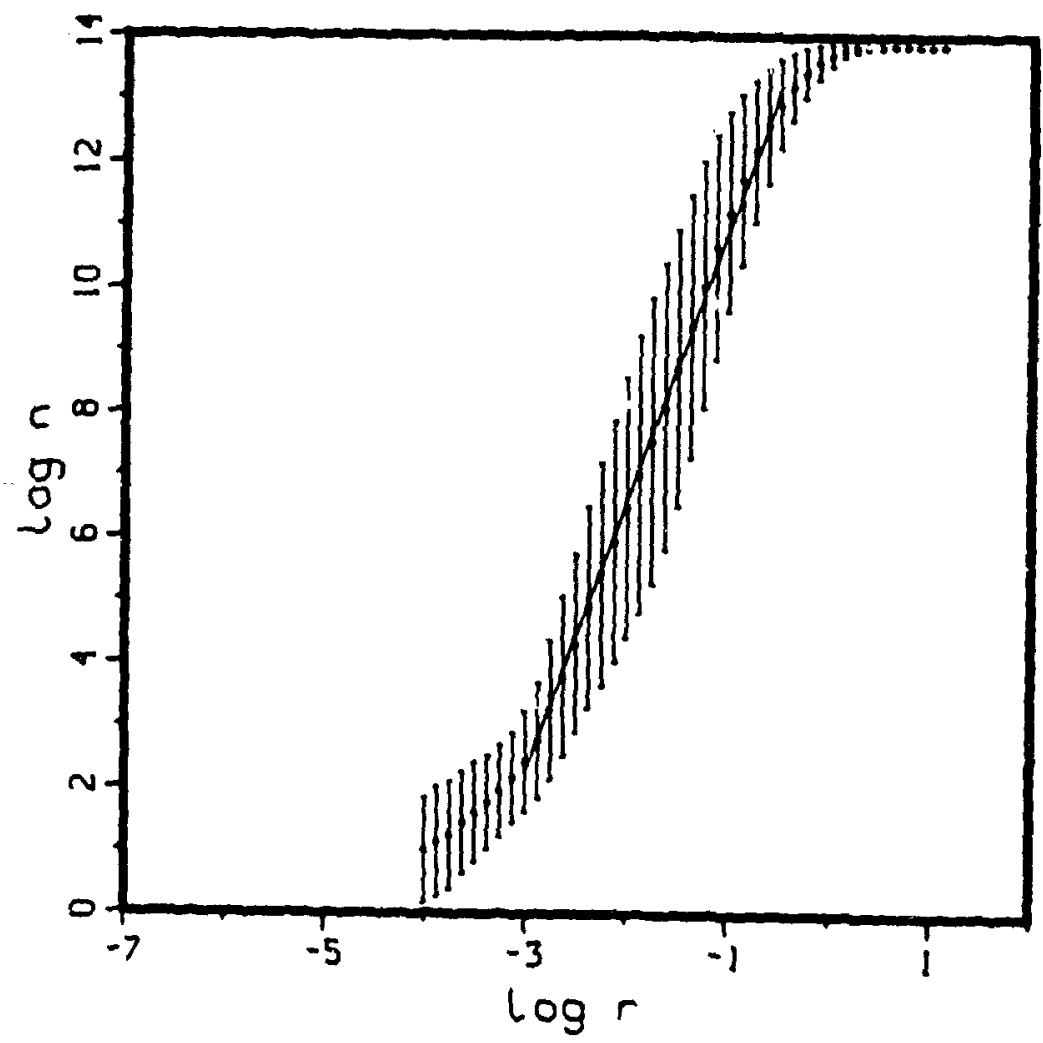

Fig. 1: "Dimension function" $\log n(r)$ vs. $\log (r)$ for lead P3-O1 while the subject was awake but quiet. We have 15,000 points at a sampling rate of $500 \mathrm{~Hz}$. Time delay: $T=20 \mathrm{msec}$, embedding dimension $N=20, n_{\text {ref }}=200$ reference points.

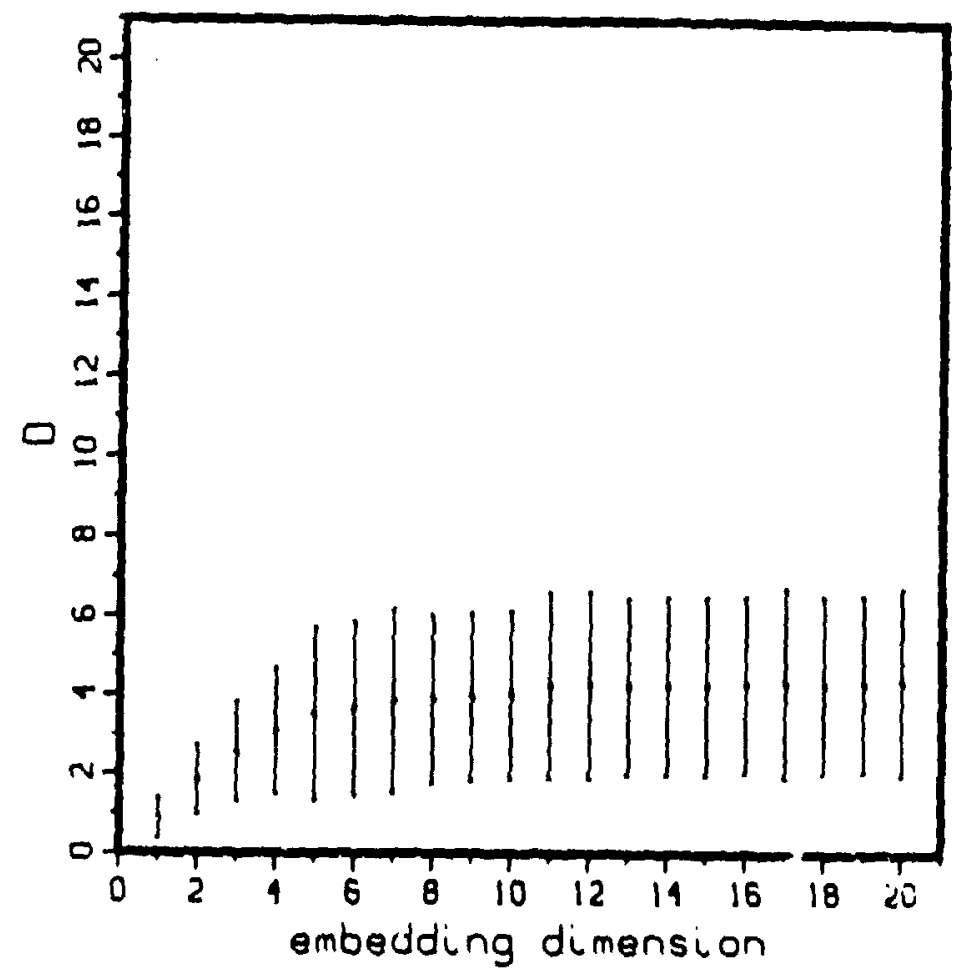




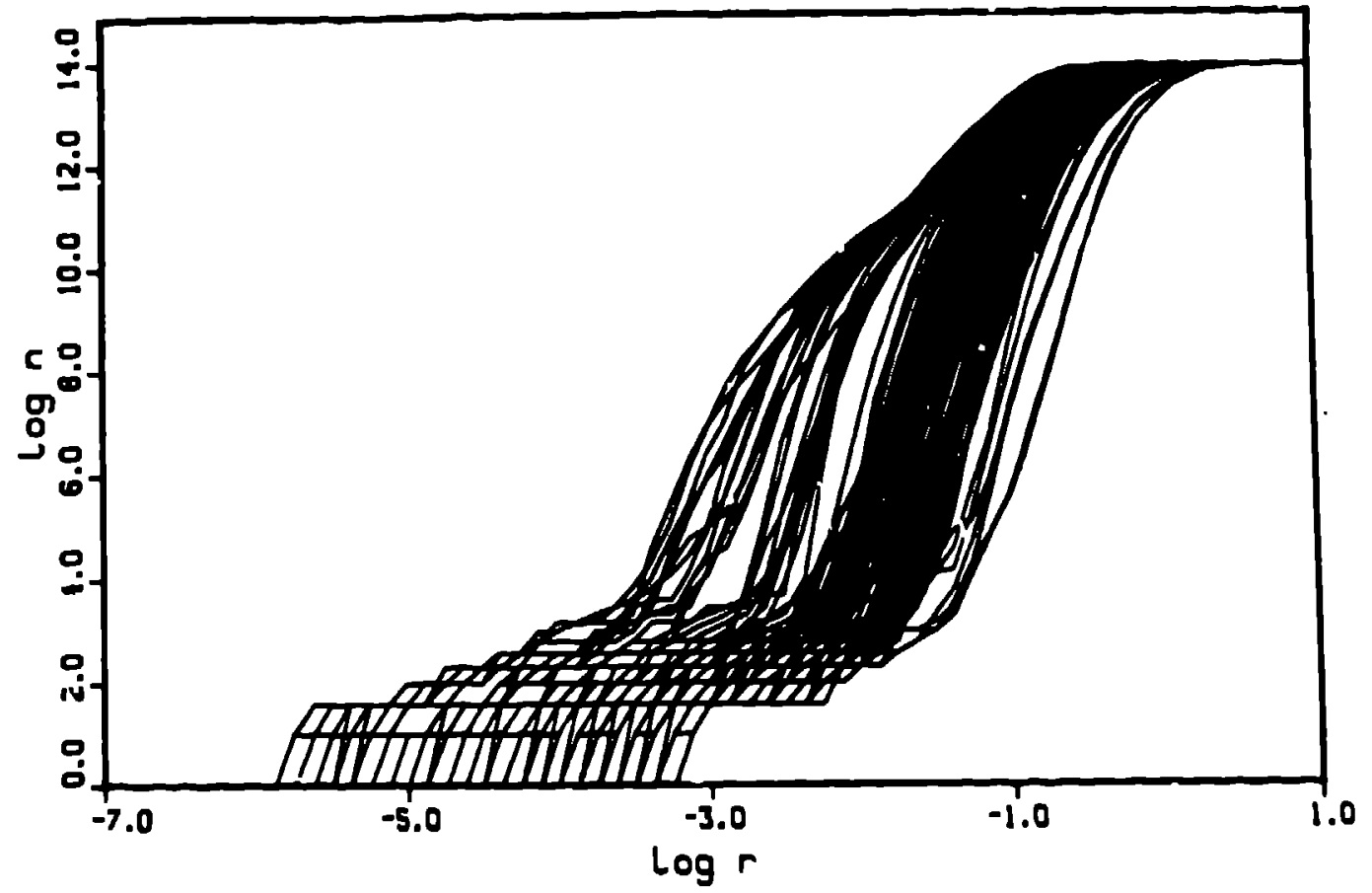

Fig. 3: Individual point-wise dimension $C_{L i v e s}$ for $n_{\mathrm{ref}}=200$ reference points. Same dats as in fig.1

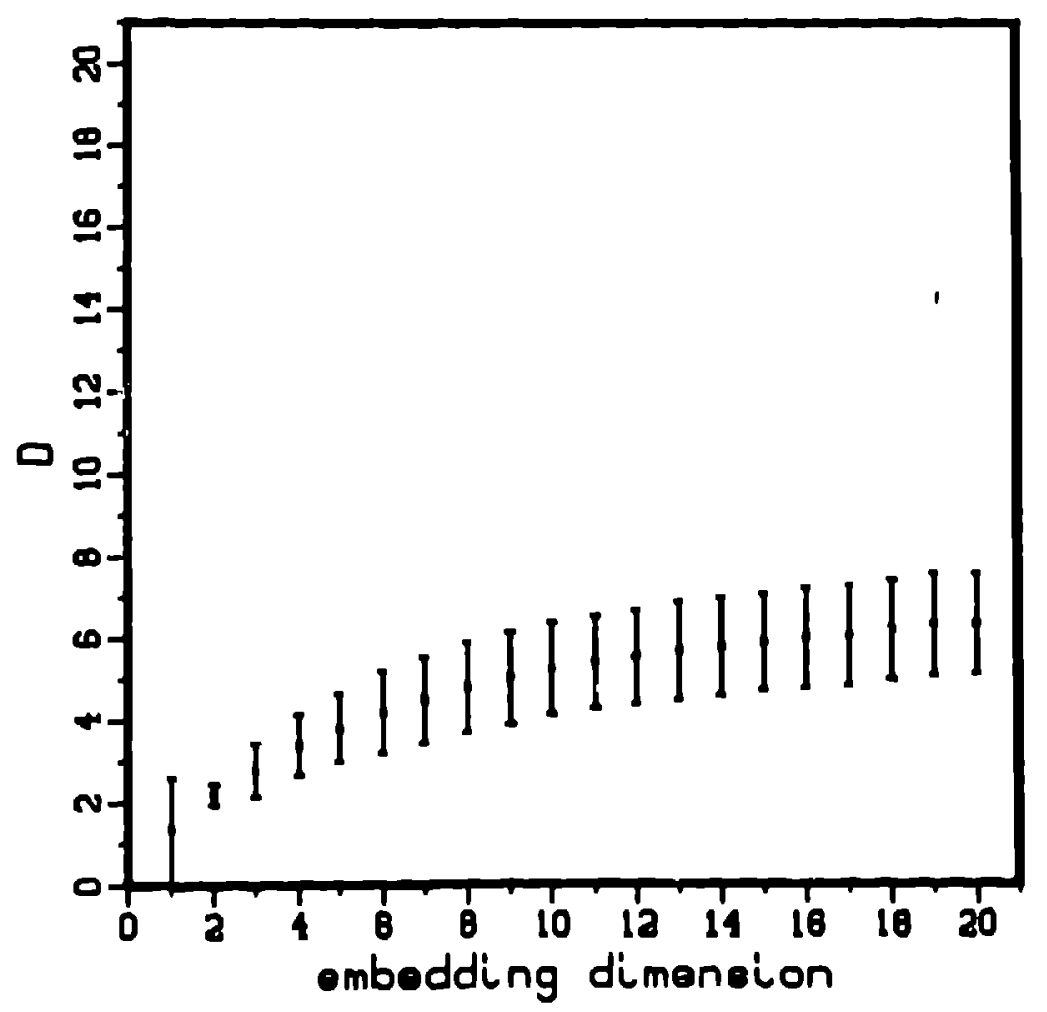

Fig. 4: Averaged point-wise dimension for increasing embedding dimension. Same data n. in $8=1$ 


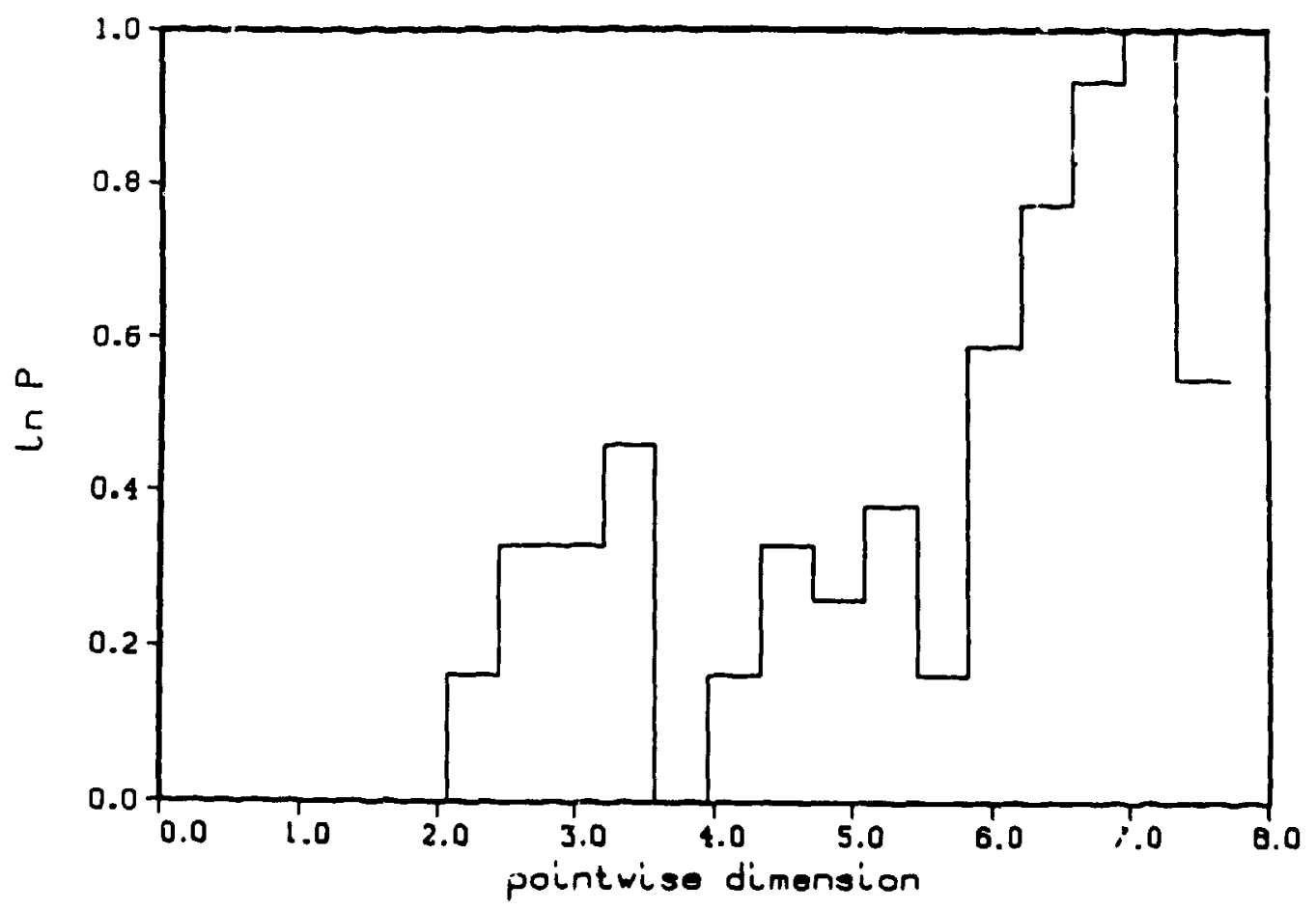

Fig. 5: Frequency of dimension values for $n_{\text {ref }}=200$ reference points

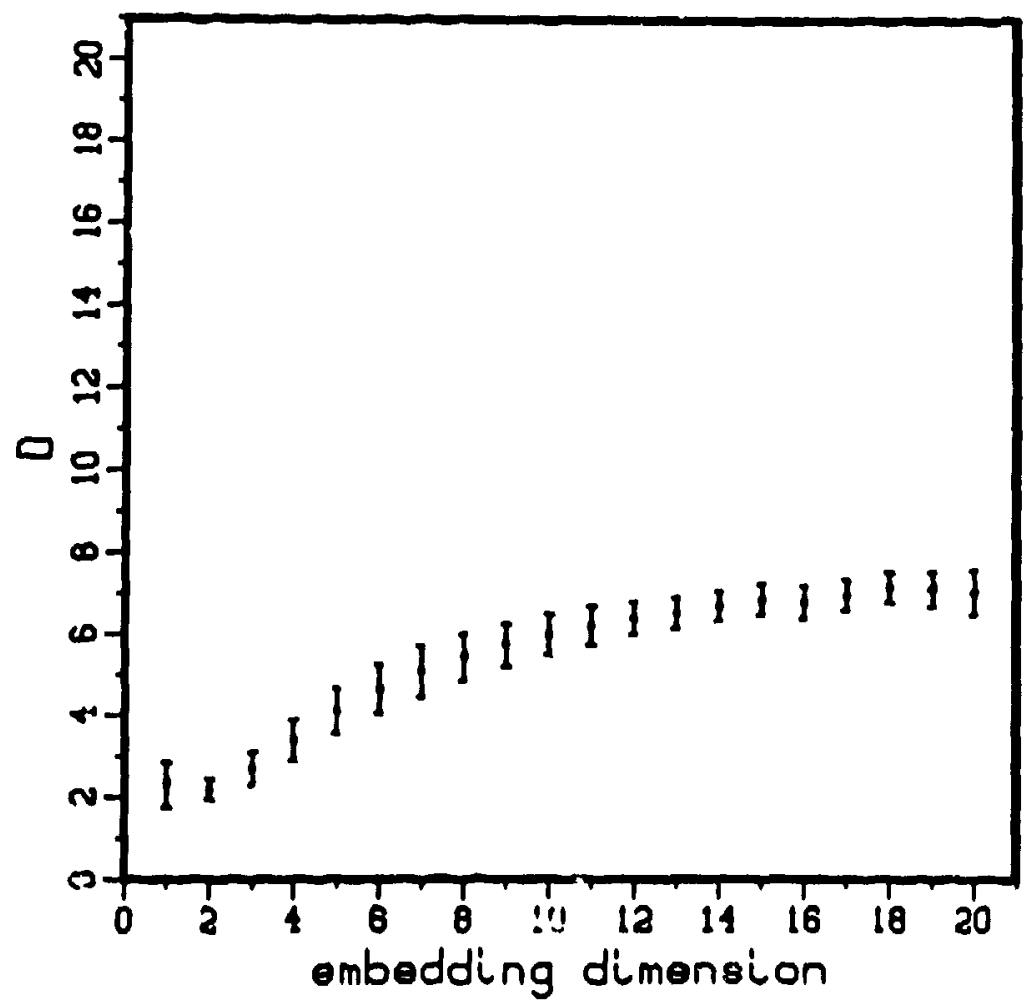

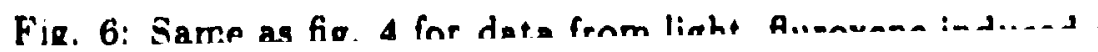


and rem sleep $\left(D^{(2)}=6.4 \pm 5.1, D^{(1)}=6.9 \pm 0.4\right)$. For stage 4 sleep we get a decrease in complexity but an increase in the fluctuation level of the information dimension $\left(D^{(2)}=5.9 \pm 4.4\right.$, $\left.D^{(1)}=6.5 \pm 0.6\right)$. Even though we thin' that these results do not correspond to the "actual dimension" of EEG in the strict sense, we believe that they show a consistent dependence on the brain state.

In the following we'd like to discuss some of the common error scurces found in dimension calculations.

Our EEG data were digitized at $500 \mathrm{~Hz}$, which is well above the maximal EEG frequency of $\leq 100 \mathrm{~Hz}$. Therefore, for very short distances $r$, neighboring vectors come from the same segment of trajectory and do not contribute to the dimensions which we want to extract. This is seen clearly in fig. 1 where the slope $d$ of the dimension curve is close to unity for $\log (r) \leq-2.5$. This effect disappears when we reduce the sampling rate to $100 \mathrm{~Hz}|3|$. Thus there is a restriction on the accuracy of the dimension algorithm: it requires a large number of data points to fill a high dimensional phase-space volume. Roughly, the number of data-points required to fill up a $N$-dimensional cube of given diameter goes exponentially with the embedding dimension $N$.

On the other hand it appears that the EEG is not stationary over the range of, say, minutes. This condition assigns an upper bound on the amount of EEG data that can be used for dimensional analysis. Generating a larger number of data points by increasing the sampling rate is not a solution, since these points are confined to low-dimensional subspaces and introduce the systematic errors described above. Therefore, we think that reports of "low-dimensional chaotic attractors" from EEG $\{17,19\}$ with dimensions less than three describe different phenomena which seems to be more related to the spectral than the dimensional properties of the system.

In order to calculate "dimension" automatically (for each embedding dimension $N$ ), we use an algorithm which fits a straight line to each point $r_{m}$ of the dimension curve 15 . Then we calculate a goodness of fit for each line segment and located the point with the best fit. This poirt defines the center of the "scaling region." Subsequently, we increase the length of the "scaling region" until the goodness of fit reaches a chosen threshcld value of $10 \%$. We also require that our algorithm does not try to fit in regions of distances for which there are too few points (bad statistics) or too many (saturation). In figs. 4-7 we have chosen $4 \leq \log _{2}(n(r)) \leq \log _{2}\left(\frac{1}{3} N_{d a t}\right)$.

Finally, for increasing values of embedding dimension $N$, the fraction of points that are close to the boundary or surface of the reconstructed data sat increases. This is a serious limitation of the algorithm, it causes a systematic underestimate of the dimension for high values of embedding dimension [19]. We want to insure an asym.piotic regime, by using large values of embedding dimension, but we also know chas sy siematic errors due to boundary effects increase. Therefore, we consider any result of $d \geq 7$ to be indistinguishable from $d=\infty$.

\section{Lyapunov Exponents}

There are currently two basic methods available for estimating the sensitivity to an experimental system to initial conditions. For chaotic behavior it is characteristic that small perturbations get amplified exponentially fast. These growth rates of small perturbations can also be used for the quantification of the degree of temporal disorder.

In the algorithm of WOLF et al. $|20|$ one computes the behavior of orbits which are close to a given reference orbit and measures their separation rates at each points of the attractor. With this method it is possible to extract the largest Lyapur.ov exponent.

In the second method we use a combination of algorithms of ECKMANN et al.'21! and S.4.YO et al. 22.|, and try to reconstruct Jacobian matrices which yield the information about the separation of nearby orbits. In this way it is possible in principle to compute all the Lyapunov exponents, i.e. the separation or convergence rates in all phase space directions. We get a quite good convergence of the Lyapunov exponents with increasing matrix dimension. (see 23/ for a discussion and comparisor of the methuds).

The convergence or divergence rates of dynamical systems typically fluctuate to a large extent along the orbits. The Lyapunov exponents describe the average bchavior of separation or convergence. The degree of fluctuations along the attractor orbits are quantified by the non-uniformityfactor $|24|$ which corresponds to the standard deviation computed from the local divergence rates. In figs. 8,9 we have the largest Lyapunov exponents together with the non-uniformity-factor for the data sets of figs. 4-7. The separation rates are given in bits per second. Notice that the 


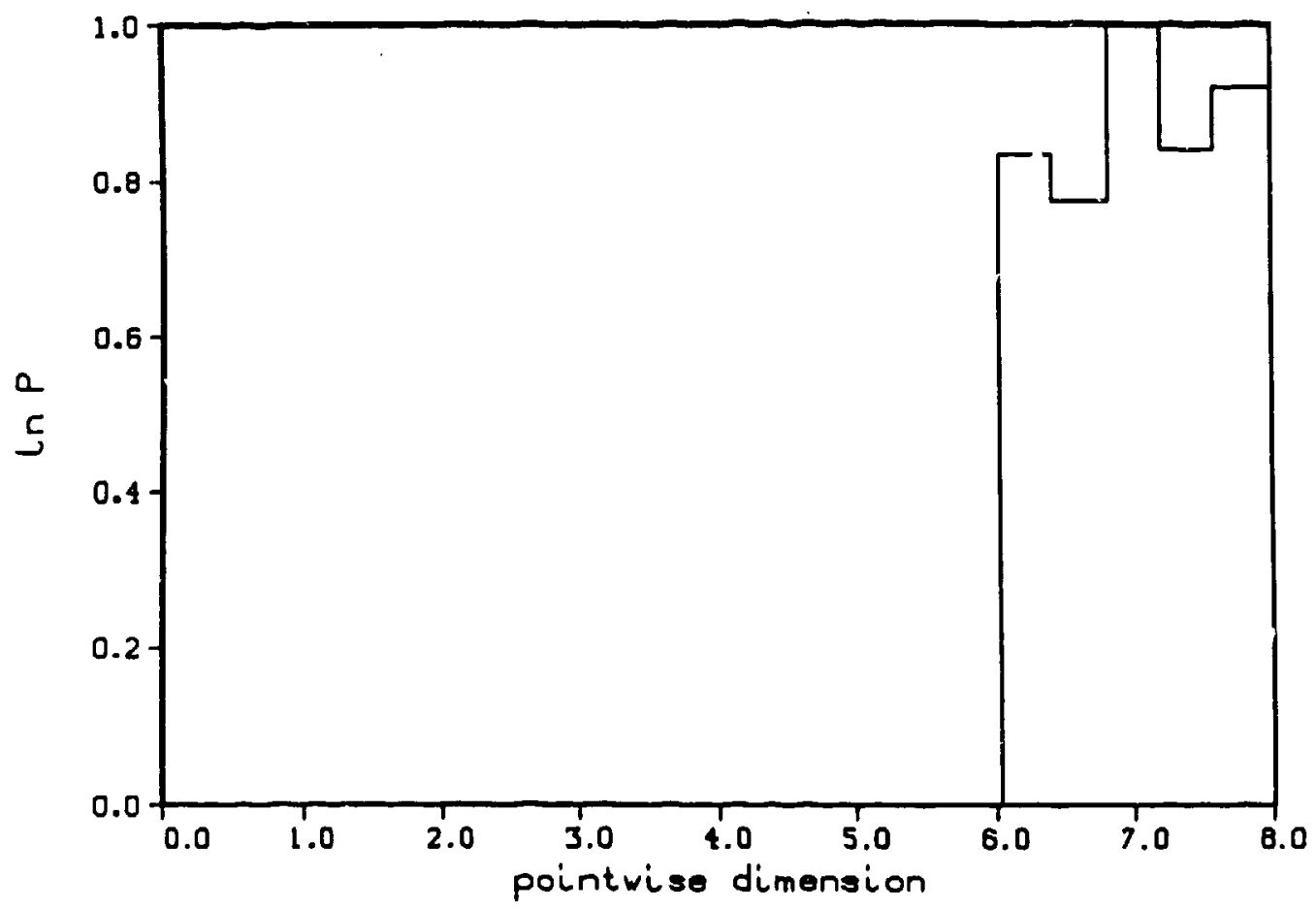

Fig. 7: Same as fig. 5 for data from light, fluroxene induced general anesthesia

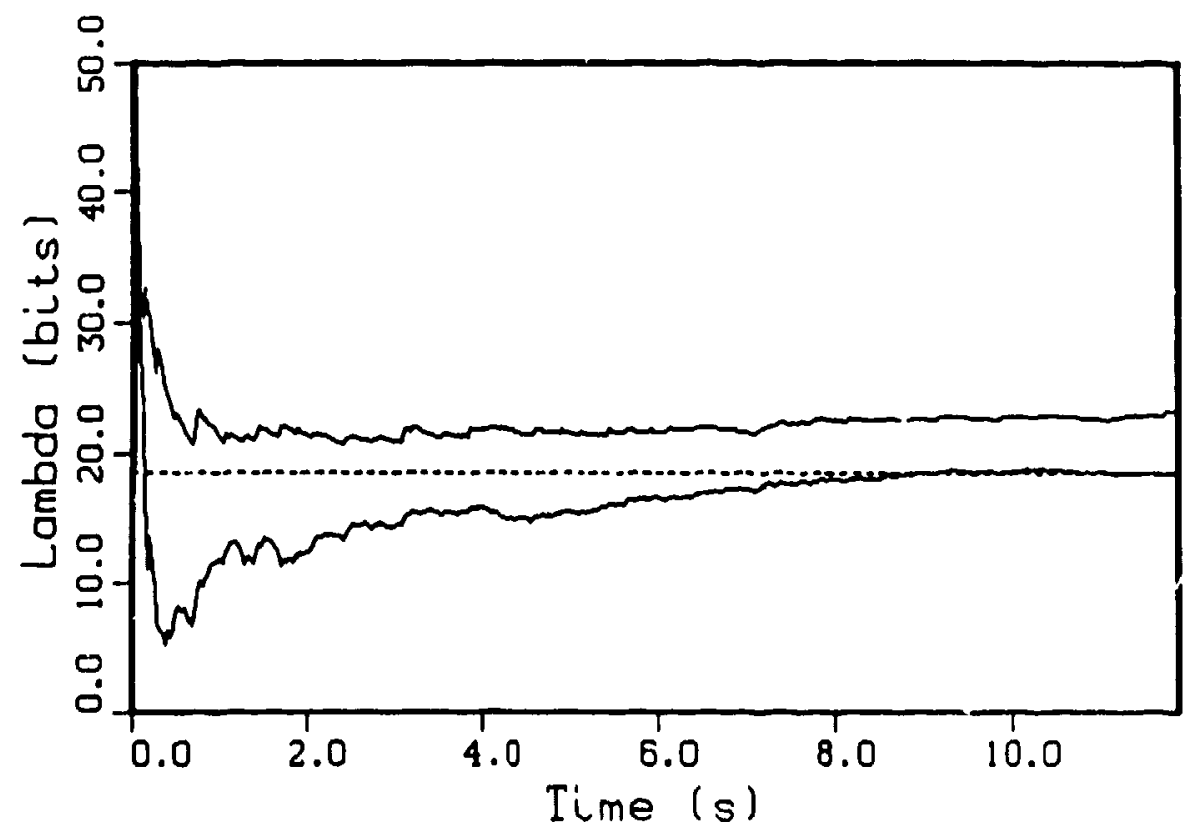

Fig. 8: Largest Lyapunov exponent according to $\mid 20\rfloor$, (lower curve), and non-uniformity

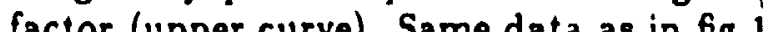




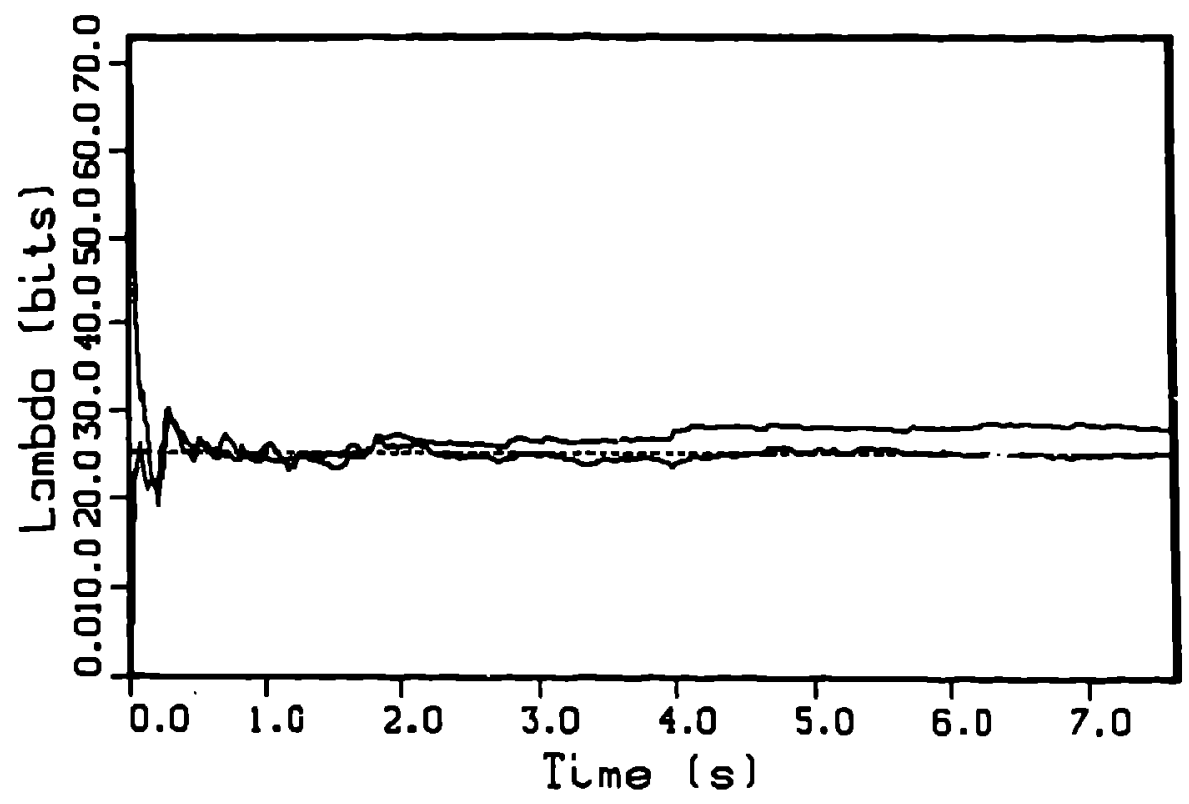

Fig. 9: Same as in fig. 8 for data of fig. 6

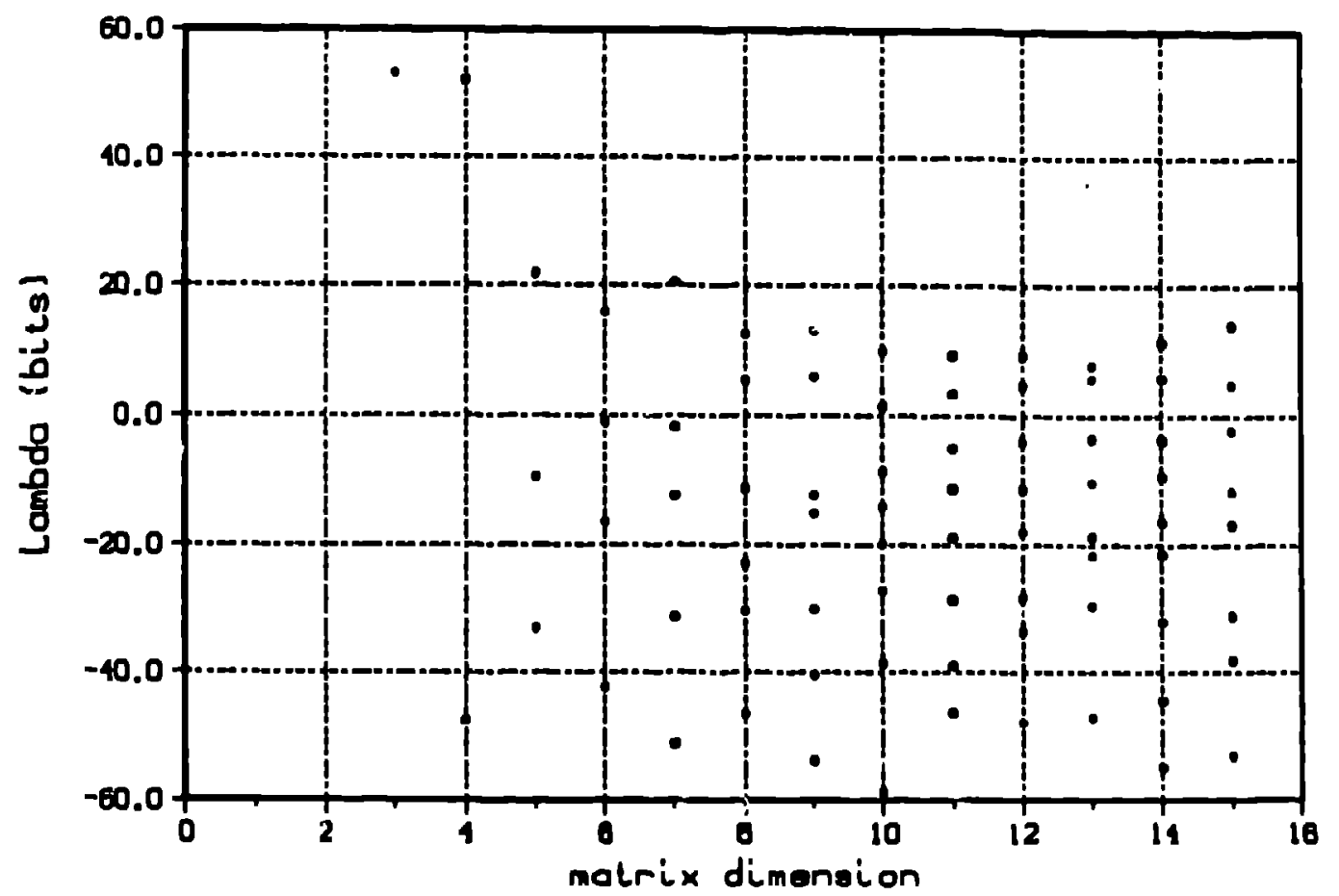

Fig. 10: The largest Lyapunov exponents according to $[21 \mid$ for increasing values of the dimension of the Jacobi-matrix. Same data as in Fig. 1. 
We can see that again in the transition to anesthesia (fig. 9) the Lyapunov exponent increases with respect to the resting awake state (fig. 8 ) while the non-uniformity-factor is reduced. This, as mentioned above, supports the interpretation that the disorder increases but simultaneously the behavior becomes more uniform.

Again some error considerations: Since for bounded attractors the separation will only be exponential for small distances, the neighboring orbits have to be reset after a short evolution time. It appears that the obtained values for the Lyapunov exponents depend on the length of the time intervals between resettings.

\section{Summary of Results and Conclusions:}

Most of our analysis was done with the awake but quiet, and anesthesia data from HANLEY [25] and data from EHLERS $\mid 26\}$, which were recorded during several stages of sleep and wakefulness.

The power spectrum of our signals (see [3]) does not show a strong power law decay. This is an additional indication that we are observing "low dimensional chaos" and not "random noise with special statistics" as discussed by OSBORNE [27].

We observo low "dimension" values, however, only in a resting state when the eyes are closed. When the subjec: opens the eyes, the dimension increases substantially. Also for the same subject, there seems to be a decrease in "dimension" for stage 4 sleep compared to the almost identical values for sleep onset and rem sleep. To our surprise we also find that the mutual information content varies significantly with different sleep stages (Fig. 27 in 3 ). In stage 4 sleep the mutual information content decays very slowly which may reflect a slow rate at which new information is produced. For the cases of REM sleep and sleep onset, the mutual information curves lie close together, which possibly reflects the similarity between the two stages of sleep [28].

During light general anesthesia induced by fluroxene the "dimelision" of leads P3-O1 and P4-O2 abruptly increase beyond our numerical limits. In the temporal leads this increase was not observed, the observed dimension is consistently very high. We don't see any changes during the transition from light to medium anesthesia. For this case, this indicates that "dimension" is not a sensitive measure of the depth of anesthesia. However, the relative increase in dimension is consistent with the excitatory action of the anesthetic fluroxene.

Finally, we want to mention that we also calculated the "dimension" of several MEG recordings [29. These data reflect activity from a smaller volume of brain tissue than EEG. Nevertheless, it seems that the dimension of these MEG data is comparable to that of the EEG data we analyzed. This might support conjectures that the electromagnetical activities in the brain are coherently generated by large regions.

ACKNOWLEDGEMENTS: We are grateful to ishn Hanley, Cindy Ehlers, and Ed Flynn for providing us with clean experimental data. One of us (G.M-K.) would like to thank Arnold Mandell, Cindy Ehlers, and Jim Havstadt for stimulating discussions.

\section{References}

1 (1) R. Elul, "The genesis of the EEG.", Int. Rev. Neurobiol. 15: 227-272, 1972

$2 \mathrm{j}$ W.R. Adey, In "Behavior and Brain Electrical Activity". X. Burcl \&. H. Altshuler, Eds.: 363-390. Plenum. New York, NY., 1974

(3) S.P. Layne, G. Mayer-Kress \& J. Holzfuss, In "Dimensions and Entropies in Chaotic Systems - Quantification of Complex Behavior", G. Mayer-Kress, Ed.: 246-256.(Springer Series in Synergetics Vol. 32). Springer-Verlag., Berlin, Heidelberg. New York. Tokyo,1986. G. MayerKress, S.P. Layne, In Proc. of the NY Acad. Sci. Conf. on Biological Dynamics and Theoretical Medicine

(4) J. Hanley, "Electroencephaiography in Psychiatric Disorders: Parts I,Il. In "Directions in Psychiatry. Vol. 4, 7: 1-8, 1984

(5) H. Haken, "Synergetics- an Introduction' (Springer Series in Synergetics Vol. 32). SpringerVerlag., Berlin, Heidelberg, New York, Tokyo,1977.

(6) M. Mackey, this volume

17) N.H. Packard, N.H., J.P.Crutchfield, J.D.Farmer, R.S.Shaw, "Geometry From a Time", Series, Phys. Rev. Lett. 45: 712, 1982

(8) A Babloyantz, this volume.

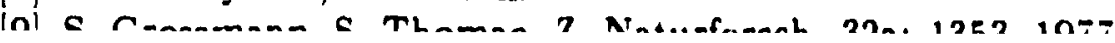


[10] R.S. Shaw, "The Dripping Faucet as a Model Chaotic System", Aerial Press. Santa Cruz, CA, 1985

(11) A.M. Fraser, H.L. Swinney, Phys. Rev. A 33: 1134-1140, 1986

12] G. Mayer-Kress, "Dimensions and Entropies in Chaotic Systems - Quantification of Complex Behavior" (Springer Series in Synergetics Vol.32) Springer-Verlag. Berlin, Heidelberg, NewYork, Tokyo, 1986

13] J.D. Farmer, E. Ott, J. A. Yorke, Physica 7D:1j3-180, 1983

14] P.Grassberger, I. Procaccia, Physica 9D: 189, 1983

15] J. Holzfusi, G.Mayer-Kress, " An Approach to Error Estimation in the Application of Dimension Algorithms", In [12]

[16] W. Lauterborn, J. Holzfuss, "Evidence for a Low-dimensional Strange Attractor in Acoustic Turbulence", Phys. Lett. 115A, 369 (1986)

17 A. Babloyantz, A. Destexhe, Proc. Nati. Acau. - Vol. 83, 3513, 1986

18] A.M. Albano, et al., in "Lasers and Brains: Conif!ex Systems with Low Dimensional Attractors", In $[12]$.

(19) J.G. Caputo, B. Malraison, P. Atten, "Determination of Attractor Dimension and Entropy for Various Flows: An Experimentalist's Viewpoint", in |12|

20] A. Wolf, J. Swift, H.L. Swinney, J. Vastano, Physica 16D, 285 (1985)

21 J.P. Eckman, D. Ruelle, Rev. Mod. Phys. 57, 617 (1985)

22 M. Sano, Y. Sawada, Phys. Rev. Let. $\underline{55}, 1082$;1385)

23] J.A. Vastano, E.J. Kostelich, " Comparison of A!gorithms for Determining Lyapunov Exponents from Experimental Data", In: |12]

24 J.S. Nicolis, G. Mayer-Kress, G. Haubs, Z. Naturforsch. 38a.115i (1983)

25 The patient was a 45 year old female. No inedication was prior to surgery. Anesthesia was induced by inhalation of fluroxene. The EEG record lasts for 15 minutes: $5 \mathrm{~min}$. awake but quiet, $5 \mathrm{~min}$. light anesthesia, and $5 \mathrm{~min}$. medium anesthesia. Analog data from the EEG was digitized at $500 \mathrm{~Hz}$.

26) Data from C. Ehlers at UCSD. The EEG data were recorded from the same subject while performing various mental tasks and while sleeping.

27) A.R. Osborne, A. Provenzale, L. Bergamasco. Preprint. Multivariate Scaling Portraits of the Lorenz Attractor.

28 A. Mandell, private communication

29) Data from E. Flynn at Los Alamos. The magneto-encephalogram data were recorded from the same subject while awake but quiet. 